\title{
The Cambrian Explosion of Popular 3D Printing
}

\author{
Juan Luis Chulilla Cano \\ CEO OnLine-Offline.net
}

\begin{abstract}
The unexpected appearance of 3D printing has caught many of technology analyst by surprise. In this paper we aim to provide a social context to the feedback loops that have generated this rapid evolution of technologies and skills involved in 3D printing, as well as and online communities related with 3D printing and the impact of this evolution on media an popular imaginary... and our near future.
\end{abstract}

Keywords - 3D Printing, Distributed Fabrication, Cambrianlike Explosion, Technological Social Enablers

\section{X.INTRODUCTION}

$\mathrm{I}$ $\mathrm{n}$ the last three years, The Internet of Things has been one of the hottest Internet topics. Every significant piece of equipment and a lot of meaningless ones is supossed that are going to receive an unique IPv6 address and means to connect to the Internet. It is a powerful forecast and, from its very beginning, a credible one: There are going to be IPv6 directions for all the imaginable beings on our planet, and connection means are less and less expensive. Meanwhile, 3D printing started to attract attention and gain momentum as 3D printer devices low both their economical and knowledge barriers. We are expecting the Internet of things, but almost nobody expected building (actual) things from the Internet.

There is an interesting connection with popular culture. While the Internet of things is a pure Internet phenomena, not predicted by speculative fiction, 3D fabrication is a recurrent topic of different sci-fi eras, with remarkables examples as Rossum's Universal Robots of Karel Čapek [1], The Second Variety of Philip K. Dick [2], The Invencible of Stanislaw Lem [3] and, of course, The Age of Diamond of Neil Stephenson [4]; In The Age of Diamond, in fact, one of the most important pieces of hardware is a universal constructor, a über3Dprinter which works on a molecular level. It doesn't matter if a future world pictured on a Science Fiction work is an Utopia or a Dystopia. What actually matters for the purposes of this paper is that main traditional Science Fiction topics are disconected to 2012 technological panorama, and main actual technological tendences of 2012 were not foreseen by sci-fi authors. We don't travel anymore to deep space, there aren't Martian or Asteroidal colonies nor interestelar travels. But Internet is ascending in its World adoption curve towards half of human population, and in our pockets are computers more powerful that the ones which sustained the Apollo program. Actually, it can be argued that as some of the bases of modern computer were ellaborated by Von Neumann in seminal works such as First Draft of a Report on the EDVAC [5] (Von Neumann:1945), it happens the same with concepts in which
3D printing roots, as we can see in Theory of Self-Reproducing Automata [6]. Both share an interesting quality of being opaque to science fiction forecasting, and both were born from theoretical seminal models down to actual operation and, after that, reshaping reality.

Indeed, there is a temporary quick\&dirty thought experiment that obtains good results: if you search for " $3 \mathrm{D}$ printing in science fiction" in Google and DuckDuckGo (hence avoiding Google's search history), the first dozens of results are related with connections between 3D printing and Science fiction. Most of these results shows 3D printing as a technology which is still coming from science fiction to reality, indeed asking to which realm 3D printing belongs. It is quite laughable that, actually, most of the Science-Fiction writers don't cover topics such as personal fabrication and selfreplication, much less as what done in (non-fictional) science. But no journalist is asking how Von Neumann ideas about universal constructors are becoming real.

3D printing outside of professional, industrial workshops and inside of classrooms, offices and even homes are a picture of a future that it is supposed to never come. While we have smartphones with gigabytes of storage capacity and multicore $\mathrm{CPU}$ of more than $1 \mathrm{Ghz}$, fabrication is supposed to continue as usual, with hordes of bluecollars mounting consumption items in a world far from our one, and not only because of geographical reasons but also because of cultural, motivational and experiencial reasons too. We are supposed to use fashionable touch interfaces, and powerful workstations, for different kind of services. It doesn't matter if such services are professional, bill-paying services or leisure ones. What really matters is that the huge majority of them are not related to physical reality. When we lecture, or program, or work with quality measurement and procedures, or evaluate, or manage, etc., we are work with people and with intangible beings, never with physical things. Things are related with lower status occupations, or with the weird and arcane activities of industrial engineers. Very few people develop hobbies related with construction, making and building, and from the very beginning of the acceptation of the term, bricolage was an activity chained to analogical means and knowledge.

We can make a double twist to the interesting social metaphor that H. G. Wells develop in The Time Machine [7]. On the one hand, the raise in the awareness of working conditions at FoxConn in the first months of 2012, after a serie of articles in NYT [8] implies a shameful echo for all of us PC and gadget users: we are the eloi who dress and uses brilliant things that are built underground, or at the other side of the world for that matter, by chinese morlocks. Those morlocks, instead of devour us, just want to survive and aspire to a consumer way of life. On the other hand, and connecting with 
Stephenson's use of morlocks and elois at In the beginning was the command line [9], most of us are elois who use computers for any kind of immaterial services, while the new morlocks are using computers, new pieces of hardware and a brand-new community knowledge for building things.

Building, in our new context, is a set of connected operations: acquire the knowledge of 3D software, of mounting a 3D printer if it came of a kit, of maintaining printer operations; design the thing we want; print it and share all the different pieces of empirical knowledge that we have been obtaining during the process in a never-ending community feedback. Moreover, these new communities are not only sharing the knowledge needed for printing and maintaining operations, but actual 3D models that can be modified and adapted, raising the Free/Open Source model of software development to a new dimension, or distributing commercial fabrication in dozens of thousands of sellers that have to compete and innovate. That is the case of these, well, 3D printer morlocks. But our elois speciation's, world vision and values have no space for 3D printing. IT effects are immaterial for the vast majority of us: we cannot touch a tweet nor taste a blog post. Moreover, as paper printers are being exiled from our offices and homes at the last part of the biblical, 40 years old journey to the Paperless office, our computers produce results that are reachable only watching a display. Most of our photos are not going to be pressed, and we are not going to save our movies in individual, physical recipients anymore. Let me insist: we elois don't touch what we do with computers.

We elois live our digital lives in a perfect, immaculate digital loop. The actual effect of this is astounding: more and more white collars develop professional activities and operations with no physical result. However, although most of us don't make things, we need, use and desire objects. On our free time, we go to all kind of shops for spending the money we earn with our immaterial services buying all kind of consumer objects.

Or maybe we should say that we went to shops. More and more people are abandoning malls, supermarkets and shops for buying online. This is not a novelty, sure, and actually the adoption curve of online shopping is well advanced in the late majority phase for most of the developed countries. Social awareness of online shopping is close to be completed.

Think about online shopping operation: we use the same device (PC) that we use for immaterial purposes, and with the same web interface we complete the transaction. Days after that, a new package is delivered to our homes or offices and we have the new acquisition in our hands.

Obviously, this is a breach of the perfect digital loop. Therefore, the cultural distance between online shopping and 3D printing is less important that it seems to be. Nowadays we all are 3D elois, but with the adequate motivation and with the unavoidable price dropping as the adoption curve of 3D printing progresses, it will be simpler and simpler to abandon the passive, consumerist attitude of only shopping online in order to build some of the products that we want or like. An adoption curve is never a matter of months, and even less with such a complex set of operations as the ones involved in 3D printing. First of all, social awareness of 3D printing is mandatory. Good and quick steps are being made in that direction, but in this year 3D printing is mostly related with technology focused people. Second, price of printers and consumables needs to drop. Droppings have been quite impressive: a factor of ten in the last 3 years. But a price $1500 \$$ and expensive materials make $3 \mathrm{D}$ printers a matter only of all kind of enthusiast, not of the general public. Finally, all the operations need to be further smothered, especially 3D designing. We have very capable 3D software since 20 years ago, both for industrial design and for animation. Indeed, too capable, too powerful for non-professionals. Although there are very powerful pieces of FOSS 3D software, such as Blender, the learning curve is quite hard for most users. Most of the 3D designing applications are not intended for 3D prototyping and/or they are quite crufty: given its degree of specialization, a good number of the components of their interfaces are comfortable for the designers only because of the prolonged use, but those interfaces are needlessly complex and non-intuitive. Keeping in mind that a mouse/keyboard combination is not intended at all for 3D operations, the only practical solution for this dilemma would be preemptive, calculating from heuristical models what the user want to do with objects and camera.

Google make a good step in the correct direction when they offered Sketchup. Although it has some quirks and idiosyncrasies, actually it lowers the learning curve and a lot of people are using Sketchup for different purposes, included 3D printing. Autodesk has recently offered 123D for free as a basic version of their famed Inventor software. It is focused on $3 \mathrm{D}$ printing and, again, learning curve is even lower than the sketchup one; moreover, it's clearly intended for most of the users who want to design 3D-printable models are not engineers trying to design complex objects. Finally, a new Finnish startup is offering a very interesting SaaS version of 3D design software, focused on 3D printing: Tinkercad. This piece of software has very elegant solutions for the problems of primitives modifications and camera movement, and it is quite close to the level of a 3D software accesible for the masses. Depending on motivation and personal approach to 3D Printing problem, there is an endless reservoir of resources for flattening adoption curve. For instance, one of the most popular 3D application, Sketchup, has a lot of tutorials available at YouTube which make it almost easy and comfortable to use. Almost. The last horse in 3D modeling, 123D, has been launched with a clear community focus, although it is not very popular for now and therefore it is not guaranteed that it is going to achieve a critical mass of users. Thingiverse is a community of builders which is growing very, very quickly in users as well as in the number of models of its library and the rest of the documentation, tutorial and general communication intended to help with a very broad range of building means, software, techniques and materials. Indeed, the raise of awareness of $3 \mathrm{D}$ printing is provoking that more and more members of the DIY online scene are paying attention to $3 \mathrm{D}$ printing and opening special sections about it, such as happens in instructables.com.

But this approach is quite connected to the "traditional" community software model. Although there are healthy and 
strong hardware development communities, the fact that hardware is physical makes more difficult the continuous, quick paced feedback between community members. However, 3D printing is so fertile for transforming ideas or software models in actual objects that it offers a strong motivation for developing together inside a community. One of the early and most impressive cases is RepRap [10]. Replicating Rapid Prototyper was in 2004 a clear departure from the previous steps in 3D building. In fact, the authors affirm that one of their inspirations was the self-reproducing automata of Von Neumann [6], which indeed closes the first grand loop of theoretical formulation / development / building-assembling. The first operational RepRap device was conceived with the objective of reproduct as many part of itself as possible, with an ambitious double objective: a) lower the costs of 3D printing as possible, in order to make it available for more and more people and b) generate as much virical effect as possible, in order to raise awareness, strong the base of a new community of builders and, even more important, extend the effects of the initiative as far as possible. While the concept is nothing sort of astounding, it has certain limitations: a) $40 \%$ percent of a RepRap machine of any iteration cannot be built by another one (metal parts, motor, circuits) b) mounting a kit is not a trivial task, especially for people without advanced knowledge. Those limitations don't permit a Cambrian explosion of 3D printing. It's very difficult to find a local, virical copy of the RepRap machine. Think about the Cambrian Explosion for a moment: before it, there were a limited number of animal phyla, but as the critical elements were finally present, Life exploded in a wave of complexity and differentiation. Not in total biomass at the very beginning, but of shapes, adaptations and ecosystem interdependencies. Precisely, Open Source License of RepRap lets other early adopters and entrepreneurs alike reinvent once and again the basic concept in new designs intended for an easier management, for even lowercost, for printing bigger or polymaterial pieces, etc. Although we are at the very beginning of a 3D printing Cambrian-like explosion, the effects are unstoppable: the old, mature players in the sector of prototyping are offering new, personal, cheaper and cheaper 3D printers. Autodesk, one of the veterans of the software part of this sector, has reacted against the unforeseen success of SketchUp for 3D modelling and has offer for free a very interesting piece of software, 123D. More and more 3Dprinting-on-demand services are competing for lowering the costs as more and more people access to 3Dprinters. What is even more impressive, there are almost mature initiatives for going beyond "traditional" 3D materials such as polilactic acid, polivinyl and ABS in order to build from metals, ceramics, etc. What was totally out of question until a couple of years ago, now it seems plausible: circuit printing.

As one of the fathers of RepRap concludes[11], our industrial fabrication model based on assembly lines for mass production has been unavoidable until now. However, 3D printing implies a truly revolutionary promise: besides having the production means for making millions of items in a few hands, we can have the production means for making a few and needed things in millions of hands. This is not fiction in the sense of a speculative writing which departs from our world, but a possible future based on developed models and current trends and events. For interstellar travel there is needed to discover totally new branches of physics; however, personal fabrication of non-complex items are a plausible extrapolation of what we know now. Indeed, we have already experienced a Digital Cambrian Explosion: what happened with computers. Before the PC was born, the computer ecosystem was very limited, with a small number of computers (compared with today's numbers) solving a comparable limited number of tasks. After all the conditions were in place (cost, operative system, killer applications, social and business awareness, the Internet), first the number of computers and after that the number of computers connected to the Internet literally exploded. Moore's law is a shallow symbol of what has happened beyond symbolic numbers such as CPU frequency, RAM amount or HD size: computers are used for a huge range of tasks and objective, both professional, communitarian, public and private. 20 years ago it wasn't foreseeable that it was going to be almost mandatory to use a PC for dealing with different public administration, that most of the companies cannot run without PCs now, that entire cultures were going to be born anew from the social web and online interaction...

We are in the glorious garage days, in which enthusiast toy and tinker with hand-made devices with an intense feeling of wonder, self-fulfilment and grasping of the future. We cannot think about 3D printing as we think about totally mature computing. It's more fair to compare near future of 3D printing with the adventing of the first PC killer applications: What are going to be the equivalents of spreadsheet and word processing software in the $3 \mathrm{D}$ printing field?

\section{REFERENCES}

[1] K. Čapek, Rossum's Universal Robots (Rossumovi univerzální roboti). English translated by David Wyllie. Adelaide: Australia:eBooks@Adelaide, $\quad 1920 . \quad$ Available at http://ebooks.adelaide.edu.au/c/capek/karel/rur/ , retrieved Feb 22, 2012

[2] P.K. Dick "The Second Variety". Space Science Fiction [UK], Volume 1 No. 5. London: The Archer Press, 1953. Available at Gutenberg Project http://www.gutenberg.org/files/32032/32032-h/32032-h.htm

[3] S. Lem, The Invincible (Niezwyciężony). London:Sidgwick and Jackson, (1962) 1973

[4] N. Stephenson, The diamond age, or, Young lady's illustrated primer. N.Y.: Bantam Books, 1995

[5] J. Von Neumann, First Draft of a Report on the EDVAC. Pa: University of Pennsylvania Press, 1945. Available at http://qss.stanford.edu/ godfrey/vonNeumann/vnedvac.pdf

[6] J. Von Neumann, and A. W. Burks, Theory of Self-Reproducing Automata. Chicago, Il.: University of Illinois Press, 1966

[7] H. G. Wells, The Time Machine. London: William Heinemann, 1895

[8] C. DuHigg and D. Barboza, D. "In China, Human Costs Are Built Into an iPad". New York Times, January 25, 2012. Available at http://www.nytimes.com/2012/01/26/business/ieconomy-apples-ipadand-the-human-costs-for-workers-in-china.html, retrieved Feb 24, 2012

[9] N. Stephenson, In The Beginning Was The Command Line. New York: Avon Books, 1999

[10] E. Sells,; Z. Smith,; S. Bailard; A. Bowyer; V. Olliver, "RepRap: The Replicating Rapid Prototyper - maximizing customizability by breeding the means of production". Handbook of Research in Mass Customization and Personalization. Bath: World Scientific, 2009.

[11] A. Bowyer, "Wealth without money". 2004. Available at http://reprap.org/wiki/BackgroundPage 